\title{
Nano-sized zeolites as modulators of thiacloprid toxicity on Chironomus riparius
}

\author{
Carla S. Lorenz ${ }^{\text {Corresp.. }}{ }^{1}$ ， Anna-Jorina Wicht ${ }^{2}$, Leyla Guluzada ${ }^{3}$ ， Barbara Crone ${ }^{4}$, Uwe Karst ${ }^{4}$, Hwa Jun Lee ${ }^{5}$, \\ Rita Triebskorn ${ }^{1,6}$, Stefan B. Haderlein ${ }^{3}$, Carolin Huhn ${ }^{2}$, Heinz-R. Köhler ${ }^{1}$ \\ 1 Institute of Evolution and Ecology, Animal Physiological Ecology, Eberhard-Karls-Universität Tübingen, Tübingen, Germany \\ 2 Institute of Physical and Theoretical Chemistry, Eberhard-Karls-Universität Tübingen, Tübingen, Germany \\ 3 Center for Applied Geosciences, Environmental Mineralogy and Chemistry, Eberhard-Karls-Universität Tübingen, Tübingen, Germany \\ 4 Institute of Inorganic and Analytical Chemistry, Westfälische Wilhelms-Universität Münster, Münster, Germany \\ 5 Center for Ordered Nanoporous Materials Synthesis, Division of Environmental Science and Engineering, Pohang University of Science and Technology, \\ Pohang, South Korea \\ 6 Steinbeis Transfer-Center for Ecotoxicology and Ecophysiology, Rottenburg, Germany \\ Corresponding Author: Carla S. Lorenz \\ Email address: lorenz.carla@web.de
}

This study investigated whether zeolites of different size (Y30 (nano-sized) and $\mathrm{H}$-Beta(OH)-III (forming large aggregates/agglomerates composed of $50 \mathrm{~nm}$ small primary particles)) exerted acute toxicity on larvae of the non-biting midge, Chironomus riparius, and whether such zeolites are able to modulate the toxicity of a common insecticide, thiacloprid, by means of adsorption of a dissolved toxicant. We conducted acute toxicity tests with fourth instar larvae of $C$. riparius. In these tests, larvae were exposed to zeolites or thiacloprid solely, or to mixtures of both compounds. The mixtures comprised $1.0 \mu \mathrm{g} / \mathrm{L}$ thiacloprid in addition to low (5.2 mg/L), medium (18.2 mg/L), and high (391.7 mg/L) zeolite concentrations, resulting in different adsorption rates of thiacloprid. As biological endpoints, changes in mortality rates and in behavior were monitored every $24 \mathrm{~h}$ over a total investigation period of $96 \mathrm{~h}$. Furthermore, we conducted chemical analyses of thiacloprid in the medium and the larvae and located the zeolite particles within the larvae by LA-ICP-MS imaging techniques. Our results demonstrate that both types of zeolites did not exert acute toxicity when applied as single-substances, but led to reduced acute toxicity of thiacloprid when applied together with thiacloprid. These results are in line with the sorption properties of zeolites indicating reduced bioavailability of thiacloprid, although our data indicate that thiacloprid can desorb from zeolites to some extent. While freely dissolved (i.e. non-sorbed) fraction of thiacloprid was a good parameter to roughly estimate toxic effects, it did not correlate with measured internal thiacloprid concentrations. Moreover, it was shown that both zeolite types were ingested by the larvae, but no indication for cellular uptake of them was found. 


\section{Nano-sized zeolites as modulators of thiacloprid toxicity on}

\section{Chironomus riparius}

\section{Authors}

4 Carla S. Lorenz ${ }^{1 *}$, Anna-Jorina Wicht ${ }^{2}$, Leyla Guluzada ${ }^{3}$, Barbara Crone ${ }^{4}$, Uwe Karst ${ }^{4}$, Hwa Jun 5 Lee ${ }^{5}$, Rita Triebskorn ${ }^{1,6}$, Stefan B. Haderlein ${ }^{3}$, Carolin Huhn², Heinz-R. Köhler ${ }^{1}$

\section{Affiliations}

$7{ }^{1}$ Institute of Evolution and Ecology, Animal Physiological Ecology, University of Tübingen, Auf der Morgenstelle 5,

8 D-72076 Tübingen, Germany

9 2Institute of Physical and Theoretical Chemistry, University of Tübingen, Auf der Morgenstelle 18 , D-72076

10 Tübingen, Germany

$11{ }^{3}$ Center for Applied Geosciences, Environmental Mineralogy and Chemistry, University of Tübingen, Hölderlinstraße

12 12, D-72074 Tübingen, Germany

13 4'Institute of Inorganic and Analytical Chemistry, University of Münster, Corrensstraße 30, D-48149 Münster,

14 Germany

$15{ }^{5}$ Center for Ordered Nanoporous Materials Synthesis, Division of Environmental Science and Engineering,

16 POSTECH, Pohang 37673, Korea

$17{ }^{6}$ Steinbeis Transfer-Center for Ecotoxicology and Ecophysiology, Blumenstr. 13, D-72108 Rottenburg, Germany

$18 *$ Corresponding author

19 Address: Institute of Evolution and Ecology, Animal Physiological Ecology, University of Tübingen, Auf der

20 Morgenstelle 5, D-72076 Tübingen, Germany

21 Phone: +49 707129 78818; E-mail: lorenz.carla@web.de 


\section{Keywords}

24 Adsorption; Chironomidae; Colloids; Nanoparticles; Pesticides; Toxicity; Zeolites

\section{Abstract}

26 This study investigated whether zeolites of different size (Y30 (nano-sized) and H-Beta(OH)-III

27 (forming large aggregates/agglomerates composed of $50 \mathrm{~nm}$ small primary particles)) exerted acute toxicity on larvae of the non-biting midge, Chironomus riparius, and whether such zeolites

29 are able to modulate the toxicity of a common insecticide, thiacloprid, by means of adsorption of a dissolved toxicant. We conducted acute toxicity tests with fourth instar larvae of C. riparius. In

31 these tests, larvae were exposed to zeolites or thiacloprid solely, or to mixtures of both compounds.

32 The mixtures comprised $1.0 \mu \mathrm{g} / \mathrm{L}$ thiacloprid in addition to low $(5.2 \mathrm{mg} / \mathrm{L})$, medium $(18.2 \mathrm{mg} / \mathrm{L})$,

33 and high $(391.7 \mathrm{mg} / \mathrm{L})$ zeolite concentrations, resulting in different adsorption rates of thiacloprid.

34 As biological endpoints, changes in mortality rates and in behavior were monitored every $24 \mathrm{~h}$

35 over a total investigation period of $96 \mathrm{~h}$. Furthermore, we conducted chemical analyses of 36 thiacloprid in the medium and the larvae and located the zeolite particles within the larvae by LA-

37 ICP-MS imaging techniques. Our results demonstrate that both types of zeolites did not exert acute

38 toxicity when applied as single-substances, but led to reduced acute toxicity of thiacloprid when 39 applied together with thiacloprid. These results are in line with the sorption properties of zeolites 40 indicating reduced bioavailability of thiacloprid, although our data indicate that thiacloprid can 41 desorb from zeolites to some extent. While freely dissolved (i.e. non-sorbed) fraction of thiacloprid 42 was a good parameter to roughly estimate toxic effects, it did not correlate with measured internal 43 thiacloprid concentrations. Moreover, it was shown that both zeolite types were ingested by the 44 larvae, but no indication for cellular uptake of them was found. 


\section{1. Introduction}

46 The toxicity of particles within the nanoscale range has been intensively investigated during the

47 last years, due to their unique properties and industrial benefits. While early studies addressed 48 "colloids" up to $1 \mu \mathrm{m}$ (Everett 1972) the focus has shifted towards "nanoparticles" with particles 49 sizes of 1-100 nm (e.g. Delay \& Frimmel 2012; Hartland et al. 2013; Lead \& Wilkinson 2006; 50 Nowack \& Bucheli 2007; Scown et al. 2010). The origin of colloids/nanoparticles can be natural 51 or synthetic (e.g. Delay \& Frimmel 2012; Lead \& Wilkinson 2006; Navarro et al. 2008; Nowack

52 \& Bucheli 2007; Oberdörster et al. 2005). Particularly synthetic particles have nowadays widespread applications, for example in electronics, personal care products, or pharmaceuticals (e.g. Handy \& Shaw 2007; Nowack \& Bucheli 2007; Oberdörster 2010; Oberdörster et al. 2005; Savolainen et al. 2010). Due to the numerous application fields, such particles enter the environment intentionally and unintentionally (e.g. Delay \& Frimmel 2012; Maynard 2006; Moore 2006; Navarro et al. 2008; Nowack \& Bucheli 2007; Wagner et al. 2014) and thus potentially may lead to detrimental effects on organisms. For example, nanoparticles were shown to cause stress in biota by inducing reactive oxygen species (Navarro et al. 2008; Savolainen et al. 2010), by affecting cell membranes (e.g. Bacchetta et al. 2012; Moore 2006; Schultz et al. 2015) or by

61 increasing the uptake of chemicals by acting as a vehicle (Moore 2006; Nowack \& Bucheli 2007;

62 Scown et al. 2010). Therefore, nanoparticles became a "hot topic" in ecotoxicology. Generally, 63 risk assessment of colloids/nanoparticles is very complex (e.g. Delay \& Frimmel 2012; Handy \& 64 Shaw 2007; Maynard 2006; Navarro et al. 2008; Oberdörster 2010; Oberdörster et al. 2005; 65 Savolainen et al. 2010), partly because adequate analytical methods to quantify nanoparticles were 66 scarce. Consequently, little is known about the environmental concentrations and fate of such 67 particles (Delay \& Frimmel 2012; Maynard 2006; Navarro et al. 2008; Oberdörster 2010; Scown 
68 et al. 2010). General predictions cannot be made, because since particles - even if chemically

69 identical - may vary in size, surface characteristics or shape, and the herewith related properties

70 (Moore 2006; Navarro et al. 2008; Oberdörster 2010; Oberdörster et al. 2005; Savolainen et al.

71 2010; Scown et al. 2010). Moreover, colloids/nanoparticles can interact with environmental

72 pollutants, other particles or biota (e.g. Delay \& Frimmel 2012; Lead \& Wilkinson 2006; Navarro

73 et al. 2008; Nowack \& Bucheli 2007; Savolainen et al. 2010; Scown et al. 2010).

74 In our study, we focused on the role of colloids/nanoparticles as sorbents for toxic chemicals.

75 While adsorption to nanoparticles could lead to enhanced exposure and thus toxicity of chemicals,

76 e.g. by colloid-facilitated transport into organisms, adsorption might also cause reduced

77 bioavailability and thus decrease the toxicity of chemicals (Baun et al. 2008; Hartland et al. 2013;

78 Lead \& Wilkinson 2006; Navarro et al. 2008; Nowack \& Bucheli 2007). In our study, we

79 investigated the effects of adsorption on the toxicity of the neonicotinoid insecticide thiacloprid in

80 the presence of nano-sized zeolites as sorbents. We tested two zeolite types with different particle

81 sizes because the size of the adsorbent might influence the effective toxicity of the adsorbate.

82 Zeolites are highly suitable materials to address these questions as they are strong adsorbents and

83 environmentally relevant due to applications in various fields, e.g. as catalysts in the chemical

84 industry, as odor control compounds, in (waste-)water treatment to remove organic pollutants and

85 heavy metals and as drug carriers (e.g. Braschi et al. 2010; Ellis \& Korth 1993; James \& Sampath

86 1999; Lehman \& Larsen 2014; Ötker \& Akmehmet-Balcığlu 2005; Wang \& Peng 2010; Yilmaz

$87 \&$ Müller 2009). However, toxicity studies so far mainly focused on micro-sized zeolites, whereas

88 studies on nano-zeolites are scarce (Lehman \& Larsen 2014). Consequently, in this study we

89 focused on the acute toxicity of nano-sized, highly sorptive zeolites (Y30 and H-Beta(OH)-III) in

90 the presence and absence of thiacloprid. The tests for acute toxicity were performed with fourth 
91 instar larvae of the non-biting midge Chironomus riparius in aqueous microcosms with exposure

92 to either suspended zeolites or dissolved thiacloprid or both. The major questions addressed in our

93 study were:

94 (1) Do zeolites per se affect the mortality rate or the behavior of C. riparius larvae under acute 95 exposure?

96 (2) Do zeolites affect the toxicity of thiacloprid on larvae of $C$. riparius under acute exposure? If 97 yes, do they act as vehicles and enhance the uptake and thus the acute toxicity of thiacloprid? Or

98 is the bioavailability and, therefore, also the toxicity of thiacloprid reduced?

99 (3) Does the particle size of the zeolite sorbents matter?

\section{2. Material and Methods}

\section{$101 \quad$ 2.1. Zeolites}

102 Zeolites are microporous, crystalline aluminosilicates, abundant in numerous natural and synthetic

103 modifications with the generalized formula $\mathrm{M}_{\mathrm{x} / \mathrm{n}}\left[\left(\mathrm{AlO}_{2}\right)_{\mathrm{x}}\left(\mathrm{SiO}_{2}\right)_{\mathrm{y}}\right] \cdot \mathrm{z} \mathrm{H}_{2} \mathrm{O}(\mathrm{n}=$ charge of $\mathrm{M})$

104 (Baerlocher et al. 2001). Their framework consists of $\mathrm{AlO}_{4}{ }^{-}-$and $\mathrm{SiO}_{4}-$ tetrahedra with aluminum-

105 and silica-atoms interconnected through oxygen-bridges (Masters \& Maschmeyer 2011). Zeolites

106 show a very homogeneous pore size distribution with a high (up to $1000 \mathrm{~m}^{2} / \mathrm{g}$ ) internal pore surface

107 area. Due to their highly microporous structure and structural anionic exchange sites, zeolites serve

108 as cation exchangers and molecular sieves.

109 The structure of the zeolite Y30 (Thermo Fisher Scientific, USA) is of faujasite type. Faujasite

110 type zeolites consist of sodalite cages and their pores are orthogonal to each-other. The surface

111 area of our $\mathrm{Y} 30$ zeolites is $780 \mathrm{~m}^{2} / \mathrm{g}$, the $\mathrm{SiO}_{2} / \mathrm{Al}_{2} \mathrm{O}_{3}$ mole ratio is $30: 1$ and the pore volume is 
$1120.56 \mathrm{~cm}^{3} / \mathrm{g}$ (based on $\mathrm{N}_{2}$ physisorption). The average pore-diameter of $\mathrm{Y}$-type zeolite is $0.74 \mathrm{~nm}$

113 and the size of a unit cell is $\sim 3 \mathrm{~nm}$, depending on $\mathrm{Si} / \mathrm{Al}$ ratio, concentration of counter-cations and

114 degree of hydration (Scherzer 1978). To obtain nano-sized particles we milled the purchased Y30

115 particles using a planetary mill (Pulverisette 5; Fritsch $\mathrm{GmbH}$ ) for $30 \mathrm{~min}$ in zirconium oxide

116 beakers using zirconium oxide milling balls of $0.1 \mathrm{~mm}$ diameter (Retsch $\mathrm{GmbH})$. After dry sieving

117 through a $0.063 \mathrm{~mm}$ grid to recover the milling balls, the milled zeolite was washed thrice in

118 distilled water and methanol and centrifuged (Herolab HiCen 21) for $10 \mathrm{~min}$ at $5000 \mathrm{rpm}$ to

119 eliminate larger particles by sedimentation. Sedimentation time and sedimentation velocity were

120 calculated using Stoke's law (Batchelor 2000).

121 Beta $(\mathrm{OH})-\mathrm{III}$ zeolites were synthesized at the Center for Ordered Nanoporous Materials Synthesis,

122 POSTECH (Korea). The reagents for the Beta(OH)-III zeolites synthesis included

123 tetraethylammonium hydroxide (TEAOH, $35 \%$ aqueous solution, Aldrich), aluminum metal (99.5

$124 \%$, Alfa Aesar), and fumed silica (100\%, Degussa). The aluminum metal source was first mixed

125 with a solution of TEAOH in water. Then, fumed silica was added to this mixture. The chemical

126 composition of the synthesis mixtures used here was $43.2 \mathrm{TEAOH} \cdot 0.8 \mathrm{Al}_{2} \mathrm{O}_{3} \cdot 80 \mathrm{SiO}_{2} \cdot 1200 \mathrm{H}_{2} \mathrm{O}$

127 (Camblor et al. 1998). After stirring at room temperature for $24 \mathrm{~h}$, the synthesis mixture was loaded

128 into a Teflon-lined $45 \mathrm{~mL}$ autoclave and heated at $140{ }^{\circ} \mathrm{C}$ for $6 \mathrm{~d}$ with agitation $(60 \mathrm{rpm})$ under

129 autogenous pressure. The solid products were then recovered by centrifugation (15,000 rpm for 10

$130 \mathrm{~min}$ ), washed repeatedly with distilled water, and dried overnight at room temperature. The

131 resulting particles with a size of $50 \mathrm{~nm}$ were calcinated in air at $500{ }^{\circ} \mathrm{C}$ for $8 \mathrm{~h}$ to remove residues

132 of the organic template $\mathrm{TEAOH}$ and were refluxed twice in $1 \mathrm{~mol} / \mathrm{L}$ ammonium nitrate aqueous

133 solution at $80{ }^{\circ} \mathrm{C}$ for $6 \mathrm{~h}$. After a secondary calcination in air at $550{ }^{\circ} \mathrm{C}$ for $2 \mathrm{~h}$ the particles $(\mathrm{H}-$

$134 \operatorname{Beta}(\mathrm{OH})-\mathrm{III})$ were obtained for further tests. 


\section{2.1.3. Size measurements and sorption experiments}

136 Particle sizes of both zeolites were measured by a laser diffraction method in aqueous suspension.

137 Zeolites were dispersed in MilliQ water (MilliQ, academic, Filter: Millipak 40 gamma gold

$1380.22 \mu \mathrm{m})$ via ultrasonication (2 min) and analyzed in a Mastersizer 2000 (Malvern Instruments)

139 with a Hydro $2000 \mathrm{~S}$ dispersing module (Malvern Instruments). We measured the particle size

140 distributions of purchased Y30 particles, milled Y30 particles, Y30 particles after milling and

141 settling, Beta(OH)-III particles and calcinated $\mathrm{H}-\mathrm{Beta}(\mathrm{OH})-\mathrm{III}$ particles. Moreover, we

142 characterized the milled Y30 particles in the supernatant after sedimentation and calcinated H-

143 Beta(OH)-III particles by SEM images using a Hitachi SU 8030 scanning electron microscope,

144 operated at an accelerated voltage of $1 \mathrm{kV}$. For this purpose, samples were sonicated in ethanol

145 before they were transferred to the surface of a silicon wafer.

146 To evaluate the sorption characteristics and to calculate the $K_{d}$ value of $Y 30$, batch experiments

147 using aqueous suspensions of zeolites and dissolved thiacloprid were conducted at controlled

148 conditions in compliance with exposure conditions of the acute toxicity test. Thiacloprid (1, 2, 4,

$1496,8,10,12,16,20,24$ and $30 \mathrm{mg} / \mathrm{L})$ and $\mathrm{Y} 30(0.1$ and $0.25 \mathrm{~g} / \mathrm{L})$ in $0.1 \mathrm{mM} \mathrm{KCl}$ were equilibrated

150 on an overhead shaker. Experiments were conducted at $\mathrm{pH} 7$ without adding buffers, and each

151 sample was prepared in triplicates. After $12 \mathrm{~h}$ of shaking, samples were centrifuged at $6240 \mathrm{~g}$

152 (Heraeus Megafuge 1.0 R Centrifuge, Thermo Fisher, at $6000 \mathrm{rpm}$ for $10 \mathrm{~min}$ ). The supernatant

153 was transferred to $1.5 \mathrm{~mL}$ autosampler glass vials and was analyzed using HPLC-UV (isocratic

154 mode; mobile phase: $\mathrm{MeOH} 60 \% / \mathrm{H}_{2} \mathrm{O} 40 \%$; retention time of thiacloprid: 5 min 36 s). To

155 determine the sorption kinetics, samples were taken and analyzed at different times (ranging from

$1561 \mathrm{~min}$ to $48 \mathrm{~h}$ ). The extent of adsorption was calculated by a mass balance based on the difference

157 of the initial and equilibrated aqueous thiacloprid concentrations. The sorption isotherms and the 
158 sorption coefficient $\left(\mathrm{K}_{\mathrm{d}}\right.$ value, which gives the ratio between sorbed thiacloprid concentration and

159 the concentration of thiacloprid in water. High $\mathrm{K}_{\mathrm{d}}$ values indicate that more thiacloprid is bound

160 to the particles, which means higher sorption) of thiacloprid at Y30 was than calculated by taking

161 an average of triplicates.

\section{2.2. Test concentrations and preparation of test solutions}

163 We chose three concentrations of Y30 zeolite (low, medium and high) to obtain 30, 60 or $97 \%$

164 sorption of the total thiacloprid, as calculated by the following equation:

$$
r_{S W}=\frac{\frac{1}{f_{W}}-1}{K_{d}}
$$

165

(eq. 1)

Where

$$
\mathrm{K}_{\mathrm{d}}-\text { distribution coefficient }
$$

170 Thus, the applied zeolite concentrations were $5.2 \mathrm{mg} / \mathrm{L}$ (low), $18.2 \mathrm{mg} / \mathrm{L}$ (medium) and

$171391.7 \mathrm{mg} / \mathrm{L}$ (high), respectively, and we used these concentrations ( $\pm 5 \%$ handling variability)

172 also for the experiments with H-Beta( $(\mathrm{OH})$-III particles. For each of the two zeolite sorbents, we

173 tested the three particle concentrations solely, as well as in mixtures with $1.0 \mu \mathrm{g} / \mathrm{L}$ thiacloprid.

174 Moreover, the effects of $1.0 \mu \mathrm{g} / \mathrm{L}$ thiacloprid solely were assessed and a control treatment with

175 filtered and dechlorinated tap water (filtered by iron and active carbon filter) was run 
176 simultaneously. Consequently, eight different treatments were tested within one experiment, with $177 \mathrm{n}=15$.

178 Test concentrations were prepared with filtered and dechlorinated tap water directly before the

179 experiment started. A stock solution of $5.0 \mathrm{mg} / \mathrm{L}$ thiacloprid (analytical standard, Sigma Aldrich,

180 Germany) in demineralized water was prepared prior to testing and was stirred overnight in the

181 dark at $7^{\circ} \mathrm{C}$. Y30 zeolites were available in an aqueous stock suspension of $2.32 \mathrm{~g} / \mathrm{L}$ zeolites

182 dispersed in aqua dest.. This stock suspension was stirred via ultrasonication (15 min) before it

183 was diluted to test concentrations. H-Beta(OH)-III zeolites suspensions were prepared directly

184 from dry zeolite powder and were dispered in the test medium by ultrasonication (15 min). After

185 setting up the test concentrations for the experiments, all dispersions were left to rest for $1 \mathrm{~h}$ to

186 allow adsorption of thiacloprid to zeolites in the mixture treatments.

\section{2.3. Maintenance of Chironomus riparius}

188 Chironomus riparius (Diptera, Chironomidae) belongs to one of the most abundant insects in

189 freshwater systems and is of high ecological relevance (Armitage et al. 1995; Langer-Jaesrich et

190 al. 2010). Larvae of this species are sediment-dwelling and feed on detritus (Armitage et al. 1995).

191 Moreover, this midge is a well-established model organism in ecotoxicology (e.g. OECD 2004a;

192 OECD 2004b; OECD 2010; OECD 2011) and toxic effects of thiacloprid on this organism have

193 been described already (Langer-Jaesrich et al. 2010; Lorenz et al. 2017).

194 The animals of our study originated from a stock culture established at our laboratory at the

195 University of Tübingen (Germany), originating from a C. riparius larvae stock obtained from

196 Goethe University Frankfurt/Main (Germany) in 2013. The animals were kept in a climate

197 chamber at a temperature of $21.0 \pm 0.5^{\circ} \mathrm{C}$ and a light-dark cycle of 16:8 h. Larvae were reared in

198 basins $(30 \times 55 \times 12 \mathrm{~cm})$, containing a $3 \mathrm{~cm}$ thick layer of fine quartz sand (particle size: $0.1-0.3$ 
$199 \mathrm{~mm}$ ) and filtered and dechlorinated tap water with a continuous, gentle aeration. They were fed 200 every second day with ground TetraMin ${ }^{\circledR}$ fish flakes (Tetra, Germany), and $50 \%$ of the water

201 volume was changed once per week. Moreover, all basins were covered by breeding cages (55 x $20265 \times 120 \mathrm{~cm}$; mesh material with a mesh size of $0.5 \mathrm{~mm}^{2}$ ) to allow swarming and mating of adult 203 midges.

\subsection{Acute toxicity test}

205 Test vessels (glass, diameter: $7.0 \mathrm{~cm}$, height: $6.5 \mathrm{~cm}$ ) were saturated overnight with the corresponding test solution, even though only low, if any, adsorption of thiacloprid on glass was expected due to the chemical properties of this compound. Earlier studies under the same experimental conditions did not reveal any adsorption of thiacloprid to glass (Lorenz et al. 2017). As glass $\left(\mathrm{SiO}_{2}\right)$ is slightly negatively charged at the $\mathrm{pH}$ applied $(7.8 \pm 0.2)$ we also exclude the zeolites (zeta potential Y30: $-42 \mathrm{mV}$; Beta(OH)-III. $-40 \mathrm{mV}$ ) to bind the surface of the vessels.

211 Subsequently, the test vessels the test vessels were emptied before $30 \mathrm{~g}$ (dry wt.) fine quartz sand

$212\left(\mathrm{SiO}_{2}\right.$, particle size: 0.1-0.3 mm; adsorption of zeolites and thiacloprid was negligible for the above-mentioned reasons) and $100 \mathrm{~mL}$ of the respective test solution were filled into them. Prior

214 to filling the vessels, all dispersions and solutions were stirred by ultrasonication for 15 min before

215 they were partitioned. So, zeolites were supposed to be equally distributed within the dispersions

216 and, consequently, also in test vessels. To adjust the temperature of the test dispersions/solutions

217 to those of the breeding basins, we incubated the test vessels for $2 \mathrm{~h}$ in the climate chamber before

218 the temperature of the medium was measured and larvae were added. Five individuals of fourth

219 instar larvae of $C$. riparius were added to each test vessel using a blunt glass pipette. The test

220 vessels were covered with perforated Parafilm ${ }^{\circledR}$ (Carl Roth GmbH, Germany) to minimize 221 evaporation and placed in a random arrangement on a desk within the climate chamber. Larvae 
222 were exposed for $96 \mathrm{~h}$ and no food was added to avoid potential interactions between food and test

223 substances. Every $24 \mathrm{~h}$ we checked the test vessels and collected data on mortality (i.e., the absence

224 of movement up to $30 \mathrm{~s}$ after gentle stimulation, or untraceable larvae) and on behavioral

225 disruption, i.e., convulsions correlated with the disability to bury themselves into the sediment.

226 Moreover, we removed dead animals to avoid contamination of the test vessels by carcass

227 decomposition products. Our criterion for test validity was a mortality rate of $\leq 10 \%$ in the control.

\section{2.5. Chemical analyses}

229 Thiacloprid analyses were conducted for water samples and larvae. The medium was analyzed to

230 determine whether actual and nominal aqueous thiacloprid concentrations matched, and to confirm

231 adsorption of thiacloprid to zeolites. For each tested concentration, one replicate was analyzed.

232 Water samples were taken from the exposure experiments directly before larvae were introduced

233 ( $0 \mathrm{~h})$ and, additionally, at the end of the exposure $(96 \mathrm{~h})$. The samples were stored in $1.5 \mathrm{~mL}$

234 Eppendorf tubes (Eppendorf, Germany) at $-18{ }^{\circ} \mathrm{C}$. Prior to analysis, samples were centrifuged at

$2353000 \mathrm{rpm}$ for $3 \mathrm{~min}$, and $1.0 \mathrm{~mL}$ supernatant was filtered through a PTFE syringe filter (pore size

$2360.45 \mu \mathrm{m}$, Chromafil, Macherey-Nagel, Germany). Thiacloprid concentrations were quantified by

237 LC-MS. Internal thiacloprid concentrations of C. riparius larvae were determined for surviving

238 individuals after termination of the exposure experiment. Animals originated from all treatments,

239 excluding those which were exposed to zeolites solely. Moreover, larvae were analyzed both with

240 gut contents (these larvae were frozen directly in liquid nitrogen after the termination of the

241 exposure) as well as with depleted guts. For gut depletion, larvae were transferred to one Petri dish

242 per treatment which was filled with $150 \mathrm{~mL}$ filtered and dechlorinated tap water after termination

243 of the exposure experiment. Petri dishes were partially wrapped in aluminum foil to reduce light-

244 induced stress (Hirabayashi \& Wotton 1998). Under these conditions, the larvae were allowed to 
245 empty their guts for $24 \mathrm{~h}$, subsequently frozen in liquid nitrogen, and stored at $-80^{\circ} \mathrm{C}$. To quantify

246 thiacloprid within the larvae, a liquid-liquid extraction method was developed based on the

247 QuEChERS extraction procedure that originally was used in food analysis but recently adjusted to

248 analyze environmental samples and biota (Anastassiades et al. 2003; Berlioz-Barbier et al. 2014).

249 For sample extraction and cleanup, 10 - $30 \mathrm{mg}$ of frozen larvae tissue was homogenized in liquid

250 nitrogen with a Micro-homogenizer (Carl Roth GmbH, Germany) in a $2.5 \mathrm{~mL}$ tubes (Eppendorf,

251 Germany). A volume of $20 \mu \mathrm{L}$ deuterated internal standard thiacloprid-D4 in methanol (Sigma-

252 Aldrich, USA) was added, resulting in a final concentration of $8.0 \mu \mathrm{g} / \mathrm{L}$ in the analyzed sample.

253 Subsequently, $0.5 \mathrm{~mL}$ water (chromasolv, Sigma-Aldrich, USA) and $0.5 \mathrm{~mL}$ acetonitrile were

254 added, and the sample was shaken for $20 \mathrm{~s}$ using a Vortex mixer. After addition of $25 \mathrm{mg} \mathrm{NaCl}$

255 (Sigma-Aldrich, USA) and $75 \mathrm{mg}$ anhydrous $\mathrm{MgSO}_{4}$ (Sigma-Aldrich, USA) the sample was

256 immediately shaken for $20 \mathrm{~s}$ using a Vortex mixer and centrifuged for $2 \mathrm{~min}$ at $10000 \mathrm{rpm}$. A

257 volume of $0.5 \mathrm{~mL}$ of the acetonitrile phase was transferred to $12 \mathrm{mg}$ primary-secondary amine

258 (PSA, Agilent technologies, Germany) and $90 \mathrm{mg}$ anhydrous $\mathrm{MgSO}_{4}$, then shaken for $20 \mathrm{~s}$ and

259 centrifuged for $2 \mathrm{~min}$ at $10000 \mathrm{rpm}$. The supernatant was concentrated in a nitrogen stream at room

260 temperature, and the residue was reconstituted in $250 \mu \mathrm{L}$ methanol and filtered with a PTFE

261 syringe filter (pore size $0.45 \mu \mathrm{m}$, Chromafil Macherey-Nagel, Germany). Also these samples were

262 analyzed by LC-MS.

263 For LC-MS analysis, a 1260 Infinity LC system coupled to a 6550 iFunnel QTOF LC/MS system

264 (Agilent Technologies, Germany) was used. Aliquots of $10 \mu \mathrm{L}$ were injected onto a Zorbax Eclipse

265 Plus C18 column (2.1 x $150 \mathrm{~mm}, 3.5$-Micron, narrow bore, Agilent Technologies, USA) with a

266 narrow bore guard column $(2.1 \times 12.5 \mathrm{~mm}, 5$-Micron, Agilent Technologies $)$. A jetstream

267 electrospray ionization (ESI) source was operated with a nebulizer pressure of $35 \mathrm{psig}$, drying gas 
268 temperature of $160^{\circ} \mathrm{C}$, a flow rate of $16 \mathrm{~L} / \mathrm{min}$, and a fragmentor voltage of $360 \mathrm{~V}$. In the positive

269 mode, capillary voltage was set to $-4000 \mathrm{~V}$, skimmer voltage to $65 \mathrm{~V}$, and the nozzle voltage to -

$270500 \mathrm{~V}$. The mass range was $80-1200 \mathrm{~m} / \mathrm{z}$ with a data acquisition rate of 1 spectrum/s. The sheath

271 gas temperature was set to $325^{\circ} \mathrm{C}$ with a flow rate of $11 \mathrm{~L} / \mathrm{min}$. For internal calibration, purine

272 and HP0921 (Agilent Technologies, USA, $\mathrm{m} / \mathrm{z}=121.0508,922.0097$ ) were used, the mass range

273 was set to $50 \mathrm{ppm}$. A gradient elution was performed at a flow rate of $0.3 \mathrm{~mL} / \mathrm{min}$ using water and

274 methanol both containing $0.1 \%$ formic acid. The initial content of $95 \%$ water was decreased after

$2751 \mathrm{~min}$ to $5 \%$ water over $7 \mathrm{~min}$ and, after another $7 \mathrm{~min}$ at $5 \%$, increased to $95 \%$ water over $30 \mathrm{~s}$.

276 Data analysis was performed with MassHunter software (Agilent Technologies, USA). For

277 quantification of water-samples, a calibration curve in the range of $0.2-10 \mu \mathrm{g} / \mathrm{L}$ thiacloprid was

278 used $\left(r^{2}=0.9991\right)$, the limit of detection was $0.2 \mu \mathrm{g} / \mathrm{L}$. Thiacloprid concentrations in larvae were

279 calculated based on peak area of the deuterated internal standard of $8.0 \mu \mathrm{g} / \mathrm{L}$ and the limit of 280 detection was $1.0 \mu \mathrm{g} / \mathrm{L}$.

\section{2.6. LA-ICP-MS imaging}

282 To verify ingestion of zeolites by the larvae, LA-ICP-MS imaging techniques for the detection of

283 aluminum within thin sections of larvae were used. Therefore, 10 surviving larvae, exposed to the

284 highest zeolite concentrations or the control, were fixed in $2 \%$ glutardialdehyde (Sigma Aldrich,

285 Germany) buffered in $0.005 \mathrm{M}$ cacodylate (sodium cacodylate trihydrate, $\mathrm{pH} 7.4$, Sigma Aldrich,

286 Germany). Fixed larvae were stored at $4{ }^{\circ} \mathrm{C}$ for at least one week. Afterwards, samples were de-

287 calcified in $5 \%$ TCA (trichloroacetic acid, $\geq 99 \%$ p.a., Carl Roth GmbH, Germany) in formol (37

$288 \%$ p.a., stabilized with methanol, Carl Roth GmbH, Germany), dehydrated with ethanol and

289 routinely processed for Technovit ${ }^{\circledR}$ embedding (Technovit ${ }^{\circledR}$ 7100, Heraeus Kulzer GmbH,

290 Germany). Embedded samples were cut by a rotation microtome (Leica RM2265, Leica 
291 Biosystems Nussloch GmbH, Germany) into sections of $7 \mu \mathrm{m}$ thickness which were used for

292 LA-ICP-MS imaging experiments. These were performed with the LA system LSX $213 \mathrm{G2}^{+}$

293 (CETAC Technologies, USA) incorporating a frequency quintupled Nd:YAG laser with a 294 wavelength of $213 \mathrm{~nm}$ and equipped with Chromium 2.2 software. The laser system device was 295 coupled to a triple quadrupole based inductively coupled plasma mass spectrometer (iCap TQ, 296 Thermo Fisher Scientific, Germany). The LA parameters relating to spot size, scanning speed, 297 laser energy and carrier gas flow were optimized based on the best signal-to-noise ratio in 298 combination with highest spatial resolution. The samples were ablated using a line by line scan 299 with a laser energy density of 7,55 J/cm², $20 \mathrm{~Hz}$ laser shot frequency, $50 \mu \mathrm{m}$ spot size and $250 \mu \mathrm{m} /$ 300 s scan speed. The aerosol was transported to the ICP with a carrier gas of helium $(0.45 \mathrm{~L} / \mathrm{min})$ 301 passing the ablation chamber. For maximum sensitivity and to minimize possible interferences, 302 the measurement was performed in kinetic energy discrimination mode (KED) with helium as cell 303 gas. ${ }^{27} \mathrm{Al}$ was monitored with dwell times of $0.1 \mathrm{~s}$. Data evaluation was performed using the 304 software ImageJ (National Institutes of Health, USA).

\section{2.7. Data analyses}

306 A generalized linear mixed model (GLMM) with binary data distribution was used, with the Petri 307 dish identity as a random factor. The statistics were done with " $\mathrm{R}$ " (R Development Core Team 308 2017) and an $\alpha$ - level set to 0.05 . SigmaPlot 13 (Systat Software) was used to plot graphs. 
309 3. Results

310 3.1. Zeolite characteristics

311 The results of the particle size measurements by laser diffraction in the aqueous phase are shown

312 in Table 1. As expected, the sizes of Y30 particles decreased with the processing of the particles

313 and matched well with the SEM results (Figure 1 A). The measured particle size of Beta(OH)-III

314 zeolites, however, was several micrometers. Although calcination reduced the particle size or

315 aggregation/agglomeration, the particles were much larger than $50 \mathrm{~nm}$, the reported particle size

316 of $\operatorname{Beta}(\mathrm{OH})-\mathrm{III}$ zeolite primary particles.

317 The comparison of $\mathrm{Y} 30$ and $\mathrm{H}-\mathrm{Beta}(\mathrm{OH})-\mathrm{III}$ zeolites in SEM measurements revealed that $\mathrm{Y} 30$

318 zeolites, processed by milling and sedimentation, formed single primary particles (Figure $1 \mathrm{~A}$ ). In

319 contrast, H-Beta(OH)-III formed much larger aggregates/agglomerates of primary particles

320 (Figure 1 B).

321 The sorption experiments showed that sorption of thiacloprid on Y30 was strong and followed the

322 Langmuir isotherm model. The $\mathrm{K}_{\mathrm{d}}$ value at low concentrations within the linear range of the

323 sorption isotherm was $82500 \mathrm{~L} / \mathrm{kg}$.

\section{3.2. Chemical analyses}

325 The chemical analyses of the medium revealed the thiacloprid concentrations did not differ 326 between the start and the end of the experiment. Moreover, it was shown that both zeolite types 327 strongly adsorbed thiacloprid. Y30 particles in the toxicity assays adsorbed thiacloprid in the

328 predicted manner, hence, the concentration of thiacloprid in mixtures was below the detection limit

$329(<0.2 \mu \mathrm{g} / \mathrm{L})$ when high Y30 concentrations were added (97\% adsorption was calculated), at 0.5

$330 \mu \mathrm{g} / \mathrm{L}$ when the medium Y30 concentration was added (60\% adsorption was calculated) and at 
$3310.65 \mu \mathrm{g} / \mathrm{L}$ for the lowest $\mathrm{Y} 30$ concentration (30\% adsorption was calculated). H-Beta(OH)-III

332 particles, however, adsorbed thiacloprid to a much higher extent, so that dissolved thiacloprid was

333 not detectable in all mixture experiments containing H-Beta(OH)-III zeolites. When applied solely,

334 the thiacloprid concentration was $1.0 \pm 0.09 \mu \mathrm{g} / \mathrm{L}$.

335 The concentrations of thiacloprid associated with larval tissue were similar for all larvae exposed

336 to thiacloprid, solely or in mixtures $(58.41 \mathrm{ng} / \mathrm{g} \pm 14.42 \mathrm{ng} / \mathrm{g}$ (wet weight) on the average),

337 irrespective of the zeolite type added or the presence of gut contents in the sample.

338

339

340

341

342

343

344

345

346

348

349

350

351

352

353

\subsection{Acute toxicity test}

In general, control animals did not show any anomalies in behavior, and the average mortality rate was $4 \pm 8 \%$ (Figure 2 and Figure 3 ). Consequently, the criterion of validity (mean mortality $\leq 10$ $\%$ in the control) was met. Moreover, both zeolites, applied as single substances, did not lead to behavioral disorders. In contrast, larvae exposed to thiacloprid as single compound or to mixtures with low or medium Y30 concentrations displayed behavioral impairments (Figure 2). However, behavioral changes were found to be delayed in a concentration-dependent manner of Y30. Moreover, larvae exposed to the treatment with the highest Y30 concentration and, thus, highest sorption of thiacloprid did not show any behavioral disruption. In the experiment with $\mathrm{H}-$ Beta(OH)-III particles, no animals with behavioral disorders were observed. Experiments with thiacloprid solely showed an almost identical increase of convulsions over time as larvae of the identical treatment type in the experiment with Y30 did. Mortality predominantly occurred between 72 and $96 \mathrm{~h}$ of exposure. In the experiment with Y30, we did not find any statistical differences between animals exposed to Y30 solely and the control (GLMM, $\mathrm{df}=3, \mathrm{~F}=0.5351$; $\mathrm{p}$ values for comparisons between the control and low Y30 concentration: 0.676, medium concentration: 0.467 , high concentration: 0.676). Nevertheless, larvae exposed to thiacloprid, 
354 solely or in the mixtures that comprised low and medium Y30 concentrations, suffered from

355 significantly increased mortality rates compared to the control or the mixture with the highest Y30

356 concentration, but were not significantly different from one another $(\mathrm{GLMM}, \mathrm{df}=4, \mathrm{~F}=10.849$;

357 p-values for the comparison between the control and thiacloprid solely, mix low and mix medium:

$358<0.001$, mix high: 0.4162; p-values for the comparisons between thiacloprid solely and mix low:

359 0.4314, mix medium: 0.0819, mix high: $<0.001 ; \mathrm{p}$-values for the comparison between mix high

360 and mix low, mix medium and thiacloprid solely: < 0.001). However, the mean mortality rate

361 decreased with increasing zeolite concentration (Figure 3 A). Larvae exposed to the mixture with

362 the highest Y30 concentration showed a low mortality rate that did not differ to the control.

363 Likewise, no statistical differences between animals solely exposed to $\mathrm{H}-\mathrm{Beta}(\mathrm{OH})$-III zeolites and

364 the control were found (GLMM, $\mathrm{df}=3, \mathrm{~F}=0.3354$; $\mathrm{p}$ values for comparisons between the control

365 and low H-Beta-OH-III concentration: 1.0; medium concentration: 0.651 , high concentration:

366 0.414). In further analyses, it was shown that the mortality rate of larvae exposed to the mixtures

367 of thiacloprid and $\mathrm{H}-\mathrm{Beta}(\mathrm{OH})$-III did not differ from the control as well. In the contrary, animals

368 exposed solely to $1.0 \mu \mathrm{g} / \mathrm{L}$ thiacloprid exhibited significantly higher mortality rates than larvae

369 exposed to the mixtures or the control (GLMM, df $=4, \mathrm{~F}=13.488$; $\mathrm{p}$-value for the comparison

370 between the control and thiacloprid solely: $<0.001$, mix low: 0.433 , mix medium: 0.660 and high:

3710.660 ; $p$-values for all comparisons between thiacloprid solely and all three mixtures was $<0.001$;

372 Figure $3 \mathrm{~B})$.

\section{3.4. LA-ICP-MS imaging}

374 To evaluate whether zeolites were taken up by the larvae, LA-ICP-MS imaging technology with

375 aluminum as marker for zeolites was used. The distribution of aluminum within the larvae

376 confirmed that both zeolites were ingested by the larvae and were widely present in the gut lumen 
377 (Figure 4). However, there was no indication that zeolites were taken up by the tissues of the

378 organism, not even by the resorptive gut epithelium. As expected, larvae of the control did not

379 show any aluminum-containing particles in the gut lumen or in any tissue.

\section{4. Discussion}

381 The purpose of this study was to assess the acute toxicity of two nano-zeolites and to test whether

382 adsorption of the insecticide thiacloprid to these zeolites alters its acute toxicity. Since the main

383 scope was on the possible interactions between thiacloprid and zeolites and not particularly on the

384 assessment of distinct field situations, we did not use environmentally relevant concentrations,

385 particularly for the nanoparticles.

386 Our results showed that both nano-zeolites, even in the rather high test concentration, did not affect

387 the chosen endpoints when applied solely. This is in accordance with published toxicity studies on

388 larger zeolites, for which save applications (e.g. in water remediation) have been shown (Lehman

$389 \&$ Larsen 2014). Unfortunately, and contrary to our expectations, H-Beta(OH)-III particles could

390 not be tested in their nano-sized form, since the small primary particles formed rather large

391 aggregates/agglomerates in aqueous dispersions. Such formation of aggregations or

392 agglomerations is a common issue in various studies dealing with colloids/nanoparticles and thus

393 an additional challenge in exposure assessment (Savolainen et al. 2010; Schultz et al. 2015). In our

394 case, we additionally expect that the H-Beta( $(\mathrm{OH})-\mathrm{III}$ aggregates/agglomerates increased in size

395 with time of exposure and thus assume that larvae were gradually exposed to smaller

396 aggregates/agglomerates during the beginning of the experiment (due to previous ultrasonication)

397 and to larger aggregates/agglomerates at the end of the exposure phase. Consequently, larvae have

398 to be considered to be exposed to varying sizes of $\mathrm{H}-\mathrm{Beta}(\mathrm{OH})-\mathrm{III}$ particles with time, but some 
399 of these particles were neither nanoparticles, nor colloids. Consequently, since the size of primary

400 particles is less important than the sizes of the aggregations (Seipenbusch et al. 2010), it is highly

401 likely that the large sizes of the aggregates/agglomerates impaired possible cell uptake or, at least,

402 largely reduced its probability. In fact, no cellular uptake of zeolite particles was detected by LA-

403 ICP-MS, neither for nano-sized Y30 nor for the larger H-Beta(OH)-III particles, even though

404 zeolites were definitely ingested by the larvae. The ingestion of zeolites by larvae is very plausible,

405 since the zeolites deposit on the substratum - larger particle aggregates faster than smaller single

406 crystal particles (e.g. Schultz et al. 2015; Scown et al. 2010) - and thus can be incorporated by the

407 sediment-dwelling and detritus-feeding larvae of C. riparius (Armitage et al. 1995). Although we

408 found no indication that these ingested particles exerted any toxic effect the results might be

409 different under chronic exposure conditions. For example, $\mathrm{Zhu}$ et al. (2010) showed that $\mathrm{TiO}_{2}$

410 nanoparticles accumulated in the intestinal tract of Daphnia magna and these animals were unable

411 to empty their guts in the usual time. Therefore, Zhu et al. (2010) hypothesized negative impacts

412 of this nanoparticle accumulation, e.g. on growth rate or reproduction, under long-time exposure.

413 So, we cannot exclude such effect for chronically exposed chironomid larvae as well.

414 In contrast to the tested zeolites, thiacloprid, applied as a single substance, affected both

415 investigated biological endpoints significantly in both experiments. Specifically, thiacloprid led to

416 heavy convulsions of larvae (associated with the inability of burying themselves into the sediment)

417 and an increased mortality rate. Both effects, convulsions and mortality of larvae, can be assigned

418 to the mode of action of thiacloprid, which stimulates the insect's nervous system by acting as

419 agonist of the nicotinic acetylcholine receptor (Elbert et al. 2008), and have been already described

420 in former studies (e.g. Langer-Jaesrich et al. 2010). Furthermore, our results corroborate the

421 findings of a previous study by Lorenz et al. (2017), where, among others, fourth instar larvae of 
422 C. riparius where exposed to $1.0 \mu \mathrm{g} / \mathrm{L}$ thiacloprid in an identical experimental setup. However,

423 although the same thiacloprid concentration was used in the mixture experiments, we found that

424 Y30 zeolites reduced the acute toxic effects of thiacloprid in a concentration-dependent manner,

425 and that all animals exposed to mixtures including $\mathrm{H}-\mathrm{Beta}(\mathrm{OH})-\mathrm{III}$ did not show any increased

426 mortality or behavioral impairment compared to the control. Our results suggest that the

427 investigated zeolites do not act as a vehicle for transporting thiacloprid into the cells of C. riparius

428 larvae, and that the different size of the particles does not have any effect in the tested size range.

429 We furthermore conclude that the insecticide's toxicity decreased due to the lower bioavailability

430 of thiacloprid caused by adsorption, as the results of the acute toxicity tests correlate well with the

431 measured equilibrium concentrations of aqueous dissolved thiacloprid. A reduction in the toxicity

432 of chemicals caused by adsorption on particles is well documented in the literature (e.g. Knauer et

433 al. 2007; Koelmans et al. 2006). For example, Baun et al. (2008) have demonstrated that nano- $\mathrm{C}_{60}$

434 particles sorb pentachlorophenol (PCP) significantly, which leads to reduced toxicity of PCP on

435 Daphnia magna when the animals were exposed to both substances simultaneously. Furthermore,

436 several studies described adsorption of chemicals on zeolites (e.g. Braschi et al. 2010; Ellis \&

437 Korth 1993; Ötker \& Akmehmet-Balcıoğlu 2005) and, for instance, James \& Sampath (1999) have

438 reported a decrease cadmium concentrations in water and fish (Oreochromis mossambicus) in the

439 presence of zeolites. However, the mortality rate of larvae exposed to a mixture of thiacloprid and

440 medium Y30 concentrations was higher than expected on the basis of a previous study (Lorenz et

441 al. 2017). There, larvae had been exposed, amongst other concentrations, to $0.4 \mu \mathrm{g} / \mathrm{L}$ thiacloprid

442 in an identical experimental setup, which resulted in a mortality rate of approximately $13 \%$.

443 Furthermore, no behavioral impairments were observed. In the present study, medium

444 concentrations of Y30 lead to a similar aqueous thiacloprid concentration, but the mortality rate 
445 was roughly 2.5-times higher and all larvae showed abnormal behavior after $96 \mathrm{~h}$ of exposure.

446 This disparity indicates that thiacloprid might desorb from the nanoparticles to some minor extent

447 under the different physicochemical conditions in the lumen of the intestinal tract. However, the

448 amount of desorbed thiacloprid must have been too low to cause negative effects when a greater

449 portion of thiacloprid was adsorbed in the experiment with high Y30 concentrations. Our

450 conclusion that thiacloprid desorbed from zeolites in the gut lumen is also supported by the

451 chemical analyses of the larvae's internal thiacloprid concentrations. Here, similar internal

452 thiacloprid concentrations were found in all animals - irrespective of the zeolite type or

453 concentration they were exposed to and irrespective of whether they had been allowed to defecate

454 or not. In this context, we feel that further research is needed to explain this uniformity in the

455 internal pesticide concentrations, particularly since it has been shown that the toxicity of

456 thiacloprid on $C$. riparius can also be reduced/delayed by non-adsorbing particles (Lorenz et al.

457 2017).

458 In our study, we have documented the mechanistic basis for the reduced acute toxicity of 459 thiacloprid when interacting with zeolites. In the future, chronic effects of both nano-zeolites used

460 in this study should be tested to draw conclusions about their ecotoxicity. Further studies should 461 also investigate the protective effects of zeolites in environmentally relevant concentrations, even

462 though the quantification of these concentrations still remains a challenging issue. However, due

463 to the high adsorption capacity that zeolites can have, measurable effects of realistic zeolite 464 concentrations present in the environment might be likely. 


\section{5. Acknowledgements}

466 We thank Leilei Luo, Reiner Anwander, Yucang Liang, Martin Pattky, Christian Zwiener, Sylvain

467 Merel, Boris Bugsel, Klaus Roehler, Sandra Dietz, Elisabeth Früh, Sang Hyun Ahn, and Suk Bong

468 Hong for discussions, helping advice, and support. We also thank Miriam Langer for reviewing

469 this manuscript and for her constructive comments.

\section{6. References}

471 Anastassiades M, Lehotay SJ, Štajnbaher D, and Schenck FJ. 2003. Fast and easy multiresidue method employing acetonitrile extraction/partitioning and "dispersive solid-phase extraction" for the determination of pesticide residues in produce. Journal of AOAC international 86:412-431.

Armitage PD, Cranston PS, and Pinder LCV. 1995. The Chironomidae: biology and ecology of non-biting midges. London: Chapman \& Hall.

Bacchetta R, Santo N, Fascio U, Moschini E, Freddi S, Chirico G, Camatini M, and Mantecca P. 2012. Nano-sized $\mathrm{CuO}, \mathrm{TiO}_{2}$ and $\mathrm{ZnO}$ affect Xenopus laevis development. Nanotoxicology 6:381-398.

Baerlocher C, Olson D, and Meier W. 2001. Atlas of Zeolite Framework Types: Elsevier.

Batchelor GK. 2000. An introduction to fluid dynamics: Cambridge university press.

Baun A, Sørensen SN, Rasmussen R, Hartmann NB, and Koch CB. 2008. Toxicity and bioaccumulation of xenobiotic organic compounds in the presence of aqueous suspensions of aggregates of nano- $\mathrm{C}_{60}$. Aquatic Toxicology 86:379-387.

Berlioz-Barbier A, Buleté A, Faburé J, Garric J, Cren-Olivé C, and Vulliet E. 2014. Multi-residue analysis of emerging pollutants in benthic invertebrates by modified micro-quick-easy-cheap-efficientrugged-safe extraction and nanoliquid chromatography-nanospray-tandem mass spectrometry analysis. Journal of Chromatography A 1367:16-32. 
487 Braschi I, Blasioli S, Gigli L, Gessa CE, Alberti A, and Martucci A. 2010. Removal of sulfonamide antibiotics from water: Evidence of adsorption into an organophilic zeolite $\mathrm{Y}$ by its structural modifications. Journal of hazardous materials 178:218-225.

490

Camblor M, Corma A, and Valencia S. 1998. Characterization of nanocrystalline zeolite Beta. Microporous and Mesoporous Materials 25:59-74.

Delay M, and Frimmel FH. 2012. Nanoparticles in aquatic systems. Analytical and bioanalytical chemistry 402:583-592.

Elbert A, Haas M, Springer B, Thielert W, and Nauen R. 2008. Applied aspects of neonicotinoid uses in crop protection. Pest management science 64:1099-1105.

Ellis J, and Korth W. 1993. Removal of geosmin and methylisoborneol from drinking water by adsorption on ultrastable zeolite-Y. Water Research 27:535-539.

Everett D. 1972. Manual of symbols and terminology for physicochemical quantities and units, appendix II: Definitions, terminology and symbols in colloid and surface chemistry. Pure and Applied Chemistry 31:577-638.

Handy RD, and Shaw BJ. 2007. Toxic effects of nanoparticles and nanomaterials: implications for public health, risk assessment and the public perception of nanotechnology. Health, Risk \& Society 9:125-

Hirabayashi K, and Wotton RS. 1998. Organic matter processing by chironomid larvae (Diptera:

Hartland A, Lead JR, Slaveykova V, O'Carroll D, and Valsami-Jones E. 2013. The environmental 144. Chironomidae). Hydrobiologia 382:151-159.

James R, and Sampath K. 1999. Effect of zeolite on the reduction of cadmium toxicity in water and a significance of natural nanoparticles. Nature Education Knowledge 4:7.

Knauer K, Sobek A, and Bucheli TD. 2007. Reduced toxicity of diuron to the freshwater green alga Pseudokirchneriella subcapitata in the presence of black carbon. Aquatic Toxicology 83:143-148. 
513 Koelmans AA, Jonker MT, Cornelissen G, Bucheli TD, Van Noort PC, and Gustafsson Ö. 2006. Black 514 carbon: the reverse of its dark side. Chemosphere 63:365-377.

515 Langer-Jaesrich M, Köhler H-R, and Gerhardt A. 2010. Assessing toxicity of the insecticide thiacloprid on 516 Chironomus riparius (Insecta: Diptera) using multiple end points. Archives of environmental 517 contamination and toxicology 58:963-972.

518 Lead JR, and Wilkinson KJ. 2006. Aquatic colloids and nanoparticles: current knowledge and future trends. $519 \quad$ Environmental Chemistry 3:159-171.

520 Lehman SE, and Larsen SC. 2014. Zeolite and mesoporous silica nanomaterials: greener syntheses, 521 environmental applications and biological toxicity. Environmental Science: Nano 1:200-213.

522 Lorenz CS, Wicht A-J, Guluzada L, Luo L, Jäger L, Crone B, Karst U, Triebskorn R, Liang Y, Anwander 523 R, Haderlein SB, Huhn C, and Köhler H-R. 2017. Nano-sized $\mathrm{Al}_{2} \mathrm{O}_{3}$ reduces acute toxic effects of 524 thiacloprid on the non-biting midge Chironomus riparius. PloS one 12:e176356. 10.1371/journal.pone.0176356

Masters AF, and Maschmeyer T. 2011. Zeolites-From curiosity to cornerstone. Microporous and 527 Mesoporous Materials 142:423-438.

Maynard AD. 2006. Nanotechnology: assessing the risks. Nano Today 1:22-33.

Moore M. 2006. Do nanoparticles present ecotoxicological risks for the health of the aquatic environment? Environment International 32:967-976.

531 Navarro E, Baun A, Behra R, Hartmann NB, Filser J, Miao A-J, Quigg A, Santschi PH, and Sigg L. 2008. Environmental behavior and ecotoxicity of engineered nanoparticles to algae, plants, and fungi.

534 Nowack B, and Bucheli TD. 2007. Occurrence, behavior and effects of nanoparticles in the environment. Environmental pollution 150:5-22.

536 Oberdörster G. 2010. Safety assessment for nanotechnology and nanomedicine: concepts of 537 nanotoxicology. Journal of internal medicine 267:89-105. 
538 Oberdörster G, Oberdörster E, and Oberdörster J. 2005. Nanotoxicology: an emerging discipline evolving 539 from studies of ultrafine particles. Environmental health perspectives:823-839.

540 OECD. 2004a. Test No. 218: Sediment-Water Chironomid Toxicity Using Spiked Sediment. OECD 541 Guidelines for the Testing of Chemicals, Section 2. Paris: OECD Publishing.

542 OECD. 2004b. Test No. 219: Sediment-Water Chironomid Toxicity Using Spiked Water. OECD 543 Guidelines for the Testing of Chemicals, Section 2. Paris: OECD Publishing.

544 OECD. 2010. Test No. 233: Sediment-Water Chironomid Life-Cycle Toxicity Test Using Spiked Water or Spiked Sediment. OECD Guidelines for the Testing of Chemicals, Section 2. Paris: OECD Publishing.

OECD. 2011. Test No. 235: Chironomus sp., Acute Immobilisation Test. OECD Guidelines for the Testing of Chemicals, Section 2. Paris: OECD Publishing.

Ötker HM, and Akmehmet-Balcığlu I. 2005. Adsorption and degradation of enrofloxacin, a veterinary antibiotic on natural zeolite. Journal of hazardous materials 122:251-258.

R Development Core Team. 2017. R: A language and environment for statistical computing. Vienna, Austria: R Foundation for Statistical Computing.

Savolainen K, Alenius H, Norppa H, Pylkkänen L, Tuomi T, and Kasper G. 2010. Risk assessment of engineered nanomaterials and nanotechnologies - a review. Toxicology 269:92-104.

Scherzer J. 1978. Dealuminated faujasite-type structures with $\mathrm{SiO}_{2} / \mathrm{Al}_{2} \mathrm{O}_{3}$ ratios over 100. Journal of Catalysis 54:285-288.

Schultz C, Powell K, Crossley A, Jurkschat K, Kille P, Morgan AJ, Read D, Tyne W, Lahive E, Svendsen C, and Spurgeon D. 2015. Analytical approaches to support current understanding of exposure, uptake and distributions of engineered nanoparticles by aquatic and terrestrial organisms. Ecotoxicology 24:239-261. 10.1007/s10646-014-1387-3

Scown T, Van Aerle R, and Tyler C. 2010. Review: do engineered nanoparticles pose a significant threat to the aquatic environment? Critical reviews in toxicology 40:653-670. 
563 Seipenbusch M, Rothenbacher S, Kirchhoff M, Schmid H-J, Kasper G, and Weber A. 2010. Interparticle 564 forces in silica nanoparticle agglomerates. Journal of Nanoparticle Research 12:2037-2044.

565 Wagner S, Gondikas A, Neubauer E, Hofmann T, and von der Kammer F. 2014. Spot the difference: 566 engineered and natural nanoparticles in the environment—release, behavior, and fate. Angewandte 567 Chemie International Edition 53:12398-12419.

568 Wang S, and Peng Y. 2010. Natural zeolites as effective adsorbents in water and wastewater treatment. $569 \quad$ Chemical Engineering Journal 156:11-24.

570 Yilmaz B, and Müller U. 2009. Catalytic applications of zeolites in chemical industry. Topics in Catalysis $571 \quad 52: 888-895$.

572 Zhu X, Chang Y, and Chen Y. 2010. Toxicity and bioaccumulation of $\mathrm{TiO}_{2}$ nanoparticle aggregates in 573 Daphnia magna. Chemosphere 78:209-215. 


\section{Table $\mathbf{1}$ (on next page)}

Particle size distribution of differently processed $\mathrm{Y} 30$ zeolites and Beta(OH)-III zeolites, obtained by laser diffraction measurements.

$d_{10}, d_{50}$ and $d_{90}$ are particle size distribution parameters, indicating that 10,50 or $90 \%[v / v]$ of all particles were smaller or equal to the indicated size. 


\begin{tabular}{|c|c|c|c|}
\hline & $\mathbf{d}_{10}[\mathrm{~nm}]$ & $\mathbf{d}_{50}[\mathrm{~nm}]$ & $\mathbf{d}_{90}[\mathrm{~nm}]$ \\
\hline Commercial Y30 & 1,082 & 2,772 & 5,838 \\
\hline Y30 milled & 603 & 1,552 & 4,401 \\
\hline $\begin{array}{c}\text { Y30 milled, } \\
\text { supernatant after } \\
\text { settling * }\end{array}$ & 202 & 441 & 691 \\
\hline $\begin{array}{c}\text { Beta(OH)-III } \\
\text { (non-calcinated) }\end{array}$ & 3,700 & 21,500 & 108,500 \\
\hline $\begin{array}{c}\text { H-Beta(OH)-III } \\
\text { (calcinated) * }\end{array}$ & 4,100 & 9,200 & 20,900 \\
\hline
\end{tabular}

1 The asterisks (*) mark the particles which were used in the acute toxicity tests 


\section{Figure 1}

SEM images.

A) Y30 zeolites (after milling and settling) and B) calcinated $\mathrm{H}$-Beta(OH)-III zeolites.

*Note: Auto Gamma Correction was used for the image. This only affects the reviewing manuscript. See original source image if needed for review.
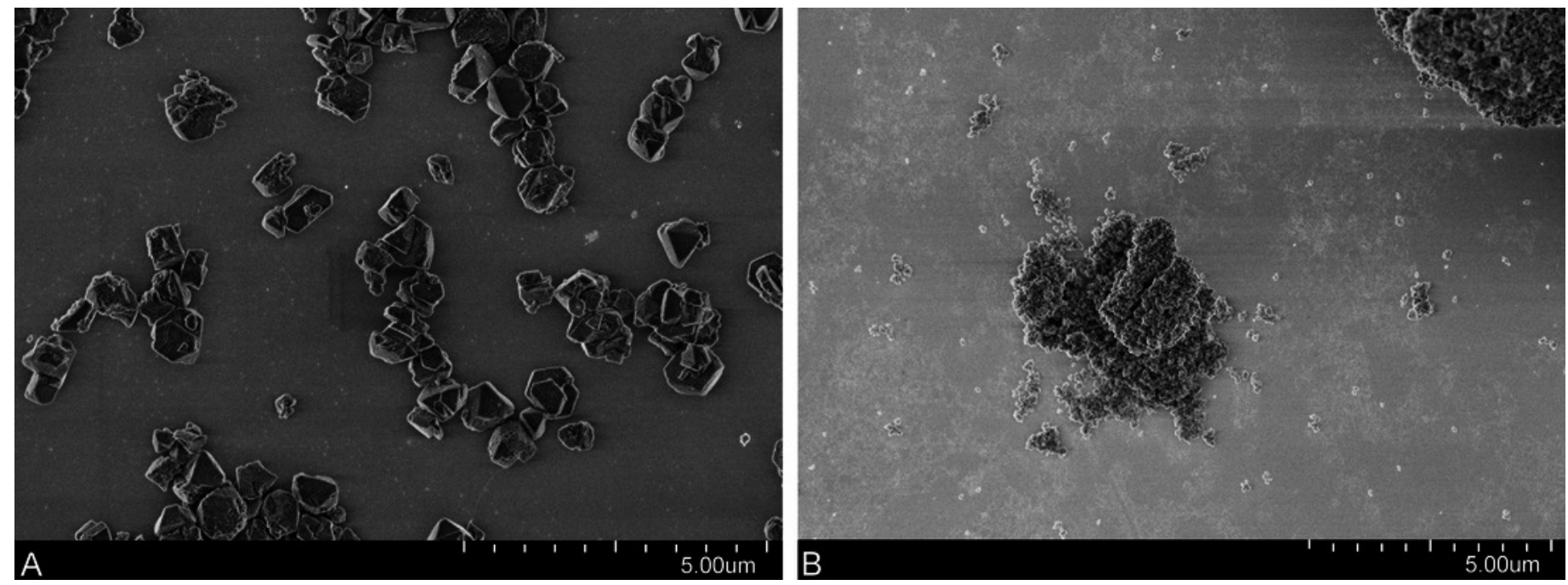
Figure 2

Behavioral disorders.

Best fitting curves, representing the percentages of living $C$. riparius larvae exhibiting convulsions in the experiment with Y30 zeolite. Animals were exposed to dechlorinated and filtered tap water as control, to $1.0 \mu \mathrm{g} / \mathrm{L}$ thiacloprid solely, or in mixtures with low, medium or high Y30 concentrations (per test vessel, $n=15$ ).

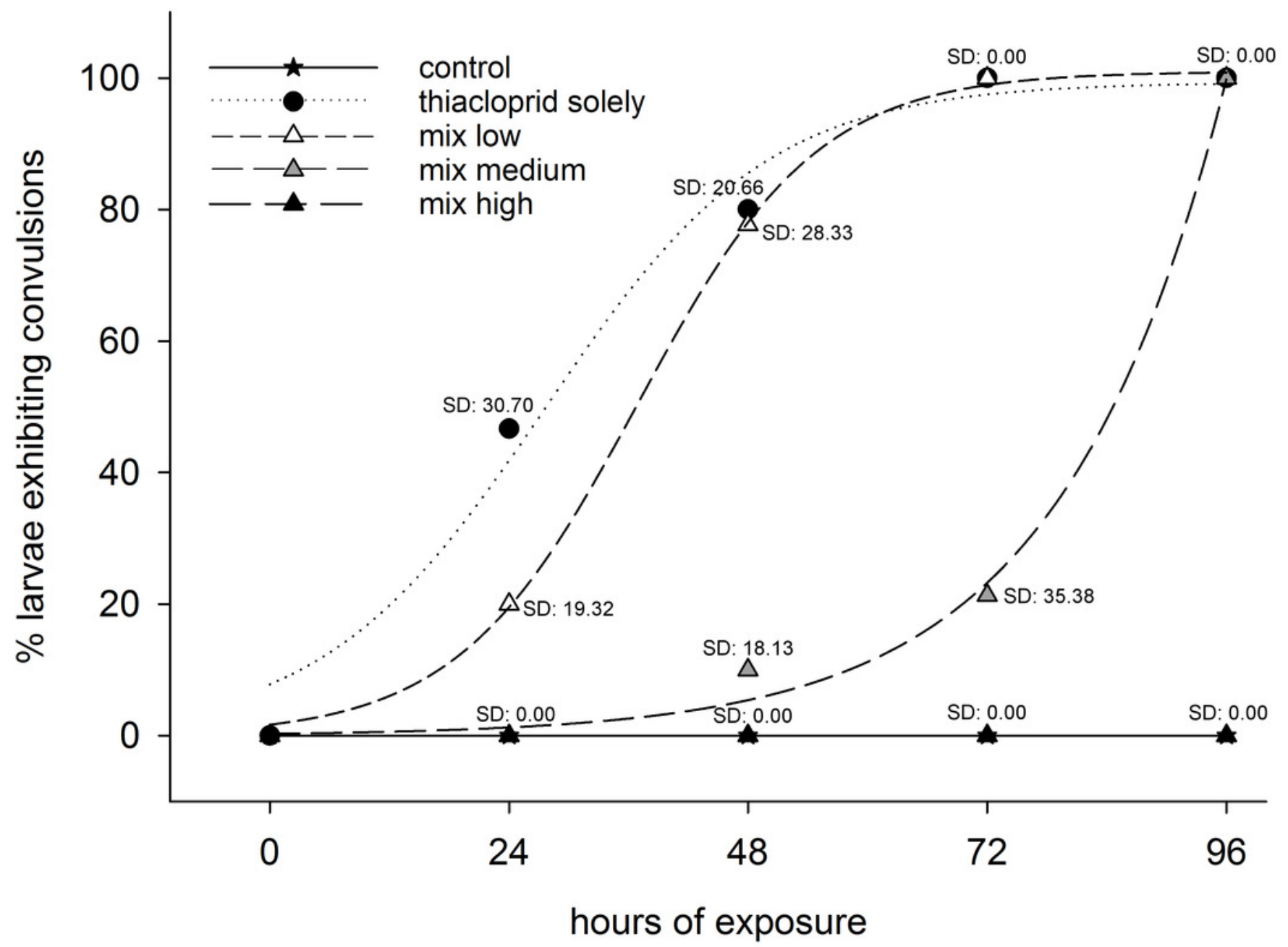




\section{Figure 3}

Mortality rates.

Means mortality rates \pm SD of $C$. riparius larvae per test vessel $(n=15)$ after exposure for 96 $\mathrm{h}$ to control conditions, to $1.0 \mu \mathrm{g} / \mathrm{L}$ thiacloprid solely, or to $1.0 \mu \mathrm{g} / \mathrm{L}$ thiacloprid in addition of low, medium and high concentrations of zeolites. A) Experiment with Y30 zeolites. Asterisks $\left.{ }^{*}\right)$ mark $\mathrm{p} \leq \alpha$. B) Experiment with $\mathrm{H}-\mathrm{Beta}(\mathrm{OH})$-III zeolites. The cross $(\dagger)$ marks the treatment group that showed significant differences vs. all other treatments. 


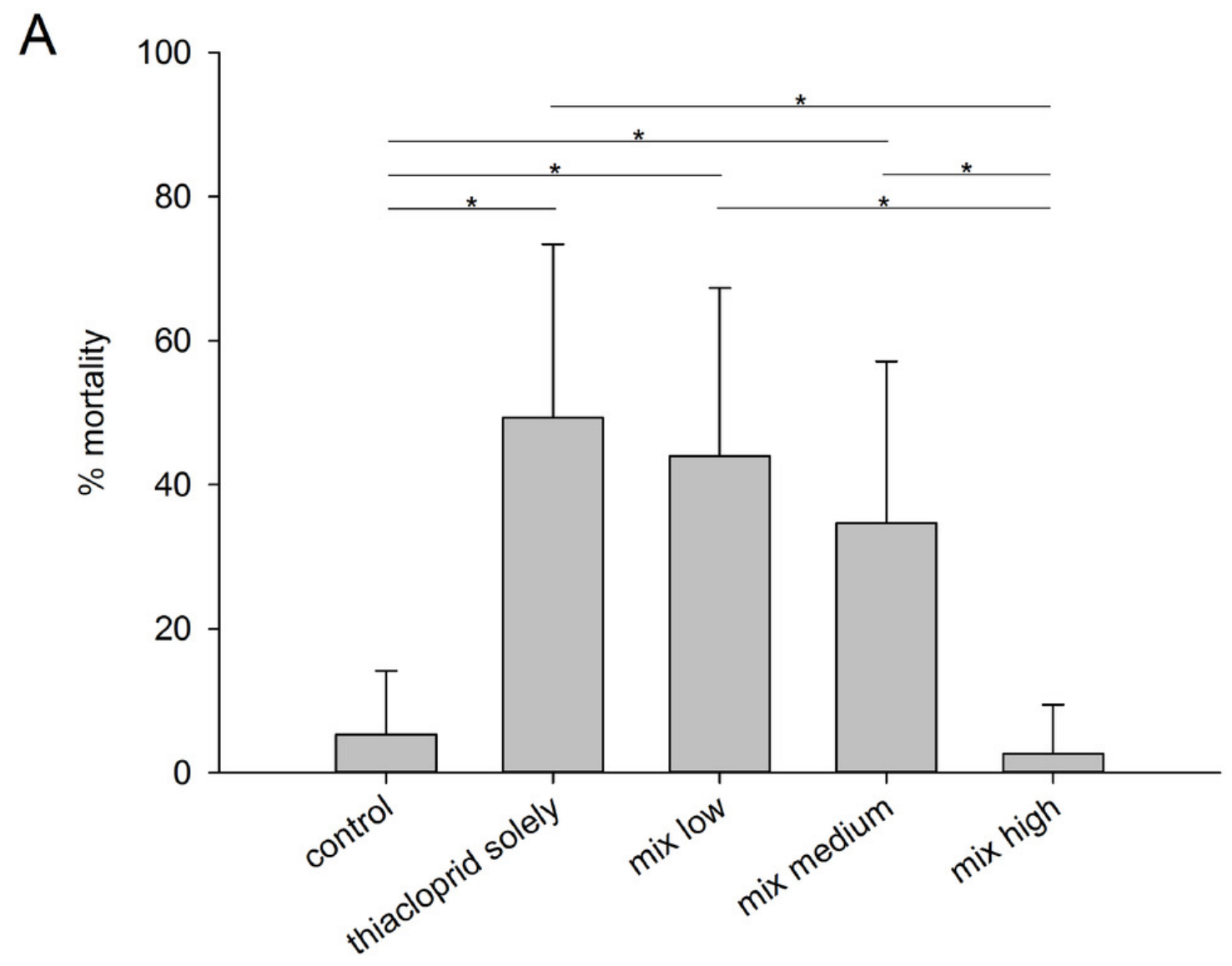

B

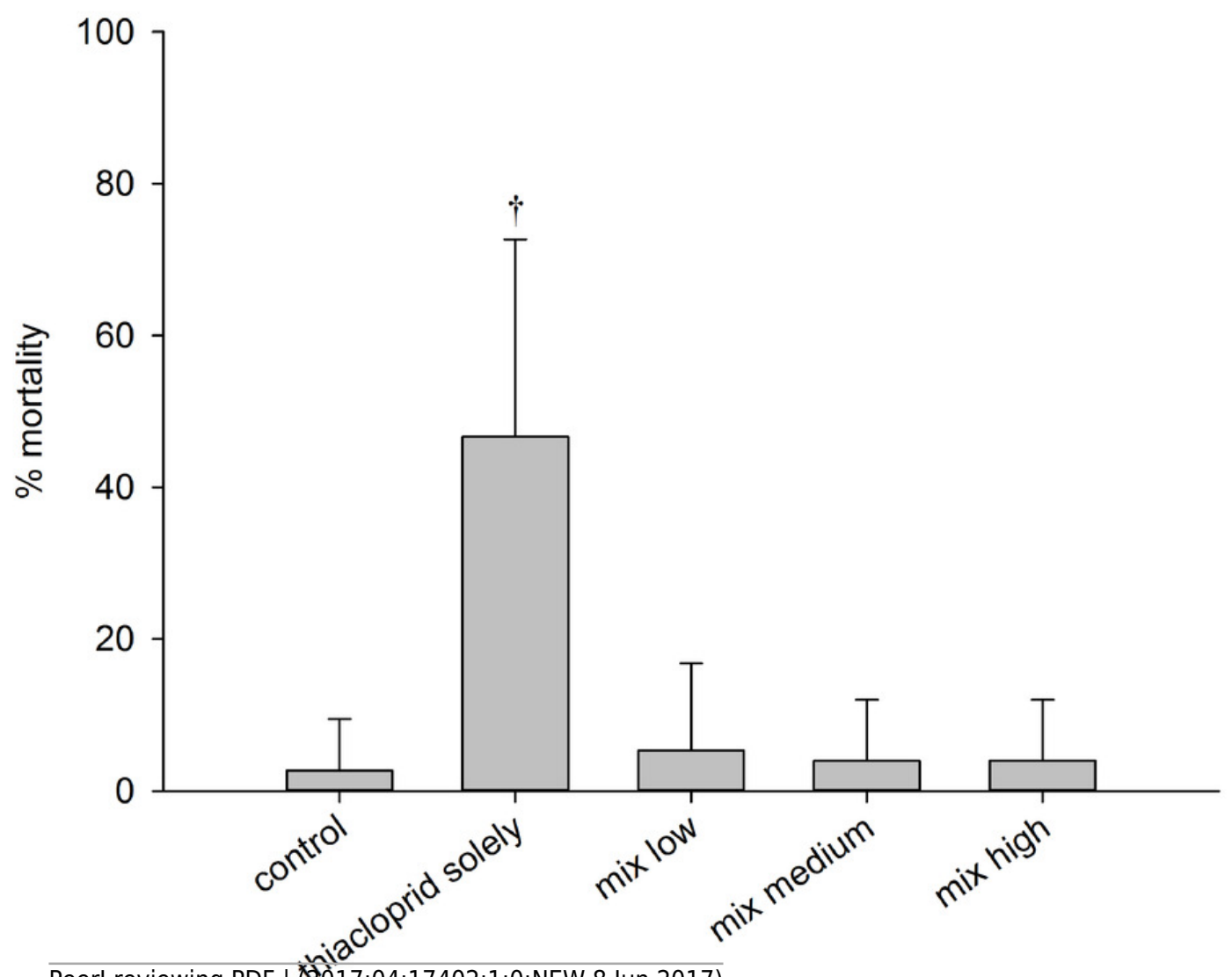




\section{Figure 4}

LA-ICP-MS analyses.

Overlay of images of a $7 \mu \mathrm{m}$ thick section of a $C$. riparius larva exposed to the highest zeolite concentration solely and LA-ICP-MS images, which show the distribution of aluminum within the larva. A) Larva exposed to $\mathrm{Y} 30$ zeolites. B) Larva exposed to $\mathrm{H}$-Beta(OH)-III zeolites.
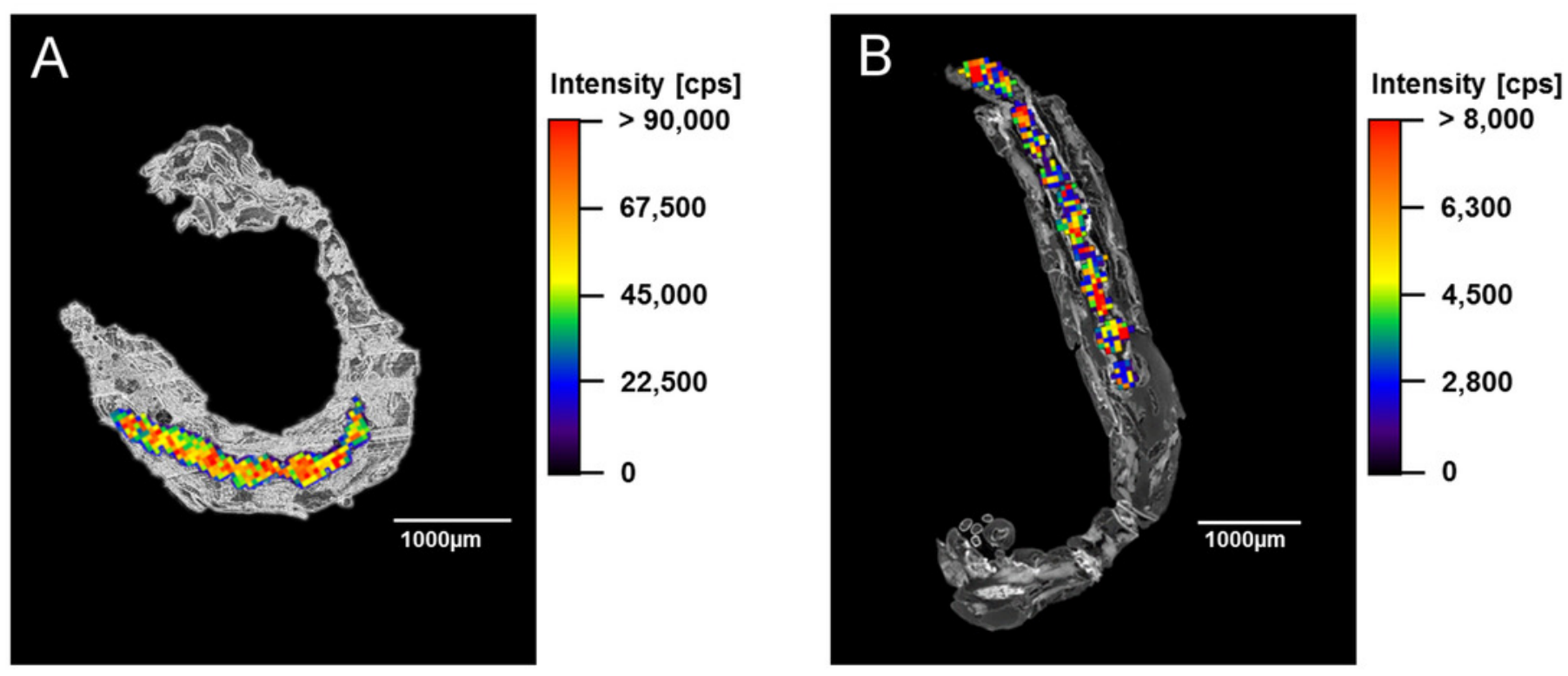